\title{
BMJ Open Comparative effectiveness and prognostic factors for outcome of surgical and non- surgical management of lumbar spinal stenosis in an elderly population: protocol for an observational study
}

To cite: Brøgger HA,

Maribo T, Christensen R, et al. Comparative effectiveness and prognostic factors for outcome of surgical and nonsurgical management of lumbar spinal stenosis in an elderly population: protocol for an observational study. BMJ Open 2018;8:e024949. doi:10.1136/ bmjopen-2018-024949

- Prepublication history for this paper is available online. To view these files, please visit the journal online (http://dx.doi. org/10.1136/bmjopen-2018024949).

Received 12 July 2018 Revised 5 October 2018 Accepted 20 November 2018

Check for updates

(C) Author(s) (or their employer(s)) 2018. Re-use permitted under CC BY-NC. No commercial re-use. See rights and permissions. Published by BMJ.

For numbered affiliations see end of article.

Correspondence to Helle Algren Brøgger; Helle.Algren.Brogger@rsyd.dk

\section{ABSTRACT}

Introduction Lumbar spinal stenosis is a common cause of low back and leg pain in the elderly and affects both physical activity and quality of life. First-line treatments are non-surgical options but if unsuccessful, surgery is advocated. The literature is not clear as to the outcome of surgery compared with non-surgical treatment, and the optimal time for surgery is not explicit. This observational study is designed to investigate the course of treatment, compare effectiveness of surgical and non-surgical management in patients with lumbar spinal stenosis and identify prognostic factors for outcome in the context of current clinical practice.

Materials and analysis Prospectively registered data on treatment, outcome and patient characteristics are collected from nationwide registers on health and social issues, a clinical registry of people with chronic back pain and hospital medical records. Primary outcome is change in physical function measured by the Zurich Claudication Questionnaire. Secondary outcomes are changes in symptom severity, pain-related function, health-related quality of life and general self-efficacy. Outcomes are assessed at baseline and 6 and 12 months. Outcomes at 12 months will be compared for patients who undergo surgery for lumbar spinal stenosis and patients managed non-surgically, using different analytical approaches. Prespecified prognostic factors of interest at baseline include treatment allocation, back and leg pain intensity, comorbidity, duration of symptoms, pretreatment function, self-rated health, income, general self-efficacy and MRIgraded severity of central stenosis.

Ethics and dissemination The study has been evaluated by the Regional Committees on Health Research for Southern Denmark (S-20172000-200) and notified to the Danish Data Protection Agency (18/22336). All participants provide consent. Findings will be disseminated in peerreviewed publications and presented at national and international conferences according to the Strengthening the Reporting of Observational Studies in Epidemiology and Prognosis Research Strategy statements. Potential sources of bias will be addressed using Risk of Bias in Non-randomised Studies of Interventions.

Trial registration number NCT03548441; Pre-results.
Strengths and limitations of this study

- The main limitation of this study is that analyses are not based on randomised treatment assignments.

- Another potential weakness may be up to $65 \%$ loss of follow-up data in the clinical registry.

- The results are strengthened by a large sample size that is 'guesstimated' from assumptions of expected patient numbers and their distribution across treatment groups.

- Data are collected prospectively and systematically as part of current clinical practice.

- Data on patient-reported outcomes are linked to data from hospital medical records and national registers on health and social issues.

\section{INTRODUCTION}

Lumbar spinal stenosis is a degenerative condition among elderly people that can substantially affect mobility, functioning and health-related quality of life (HRQL). ${ }^{1}$ The cardinal symptom of lumbar spinal stenosis is neurogenic claudication, consisting of lower limb pain and neurological symptoms exacerbated with walking and standing. ${ }^{2}$ Some degree of stenosis may be present in up to $80 \%$ of patients over 70 years old, ${ }^{3}$ but symptomatic stenosis seems relatively uncommon. ${ }^{4}$ Lumbar spinal stenosis has been reported in $13 \%-14 \%$ of patients with low back pain who see a specialist and $3 \%-4 \%$ who see a general physician. ${ }^{2}$ The natural course of lumbar spinal stenosis is largely unknown, but over the course of 10 years, symptoms have been found to worsen in $31 \%$ of patients, improve in $38 \%$ and remain unchanged in $31 \%$ despite progressive anatomic changes. ${ }^{5}$ Peer-reviewed literature does not seem to support the concern that patients managed non-surgically may be 
at risk of worsening health status over time. ${ }^{6}$ As the ageing population continues to grow, the number of individuals with lumbar spinal stenosis and the associated public health and economic consequences must be expected to increase.$^{6}$ Identifying effective treatment options for this population is thus important. ${ }^{6}$

First-line treatment is non-surgical and may include a combination of drugs, exercise, manual therapy, ${ }^{7}$ lifestyle modification and multidisciplinary rehabilitation. ${ }^{2}$ If unsuccessful, surgery is advocated. Lumbar spinal stenosis is the most frequent indication for spinal surgery in patients 65 years or older, with 3-11.5 cases per 100000 inhabitants per year. ${ }^{3}$ The literature is not clear as to the outcome of surgery compared with non-surgical treatment, ${ }^{1}$ however, and the optimal timing for surgical decompression has not yet been established. ${ }^{8}$ Due to the fluctuating natural history of lumbar spinal stenosis, surgery may be offered to patients soon after the onset of symptoms or several months or even years later. ${ }^{8}$ Surgery has been reported as the most effective treatment for lumbar spinal stenosis when outcome is defined as reduced pain and disability and improved quality of life. ${ }^{9}$ This was not the case for walking distance, however, which is the key functional limitation associated with lumbar spinal stenosis. ${ }^{9}$ A recent systematic literature review reported no differences in pain-related disability between surgery and non-surgical treatment at 3,6 and 12 months, with only one of five studies reporting a difference in favour of surgery. ${ }^{1}$ The undeniable pathoanatomical aetiology of lumbar spinal stenosis makes it understandable that some patients prefer a surgical solution, but important concerns have been raised about surgical risks, particularly for elderly patients having complex fusions. ${ }^{10} 11$ Studies have shown similar outcomes in elderly individuals undergoing minimally invasive spine surgery (without fusion) with little additional risk. ${ }^{11}{ }^{12}$ Hence, it is relevant to investigate the outcome of non-surgical treatment and to identify prognostic factors that could help determine which patients are more likely to manage without surgical treatment, and which patients would benefit more from surgery.

Multiple factors may be related to the outcome from lumbar spinal stenosis surgery including duration of symptoms, greater back pain relative to leg pain, ${ }^{13}$ smoking, ${ }^{14}$ previous spinal surgery, ${ }^{15}$ preoperative function, self-rated health, income, comorbidity and psychosocial factors. ${ }^{16}$ Self-efficacy is assumed to be an underlying factor explaining positive effects on health behaviour, health status, self-management behaviour and healthcare utilisation in older people with chronic disease ${ }^{17}$ Radiological severity on preoperative MRI is not associated with clinical severity nor surgical outcome after 1 year. ${ }^{18} 19$ Symptomatic improvement is also seen in non-surgical treatment, but evidence is sparse for prognostic factors of outcome in non-surgical management ${ }^{20}$ and the aspects that guide allocation of treatment. ${ }^{21}$

\section{Rationale for this study}

Evidence-based guidelines for clinical practice are lacking, ${ }^{1}$ and decision-making related to management of symptomatic lumbar spinal stenosis in daily clinical practice remains a challenge. ${ }^{22}$ The overall evidence from randomised controlled trials comparing surgical and non-surgical treatment is of low quality ${ }^{1}$ and provides little confidence to conclude whether a surgical or non-surgical approach is better for lumbar spinal stenosis. ${ }^{1}$ Comparative effectiveness research aims to guide decision-making by comparing the effects on clinical outcomes of a number of prespecified treatments in current use. ${ }^{23}$ The emphasis on clinical goals and decisions distinguishes comparative effectiveness research from trials that are designed to compare an experimental intervention or exposure to a control comparator (unexposed), to establish proof of concept or to elucidate a mechanism of action. For that reason, we have decided to perform an observational study in the context of clinical practice, focusing on the comparative effectiveness of surgical and non-surgical management of lumbar spinal stenosis and prognostic factors for outcome.

\section{Aim and objectives}

The aim of this study is to investigate the course of treatment for elderly patients with lumbar spinal stenosis with the objectives to (1) compare the effectiveness of spinal surgery to non-surgical management and (2) identify prognostic factors at baseline for outcome at 12 months follow-up.

\section{METHODS AND ANALYSIS \\ Design and setting}

Prospectively registered data on treatment, outcome and patient characteristics are obtained from nationwide registers on health and social issues, ${ }^{24}$ a clinical registry of people with chronic back pain (SpineData) located at the Spine Centre of Southern Denmark, Lillebaelt Hospital, Middelfart, Denmark ${ }^{25}$ and hospital medical records. Baseline data are captured at point of first clinical contact and at 6-month and 12-month follow-ups (see figure 1).

\section{Participants}

All patients older than 60 years, diagnosed with lumbar spinal stenosis, and having first clinical contact at the Spine Centre of Southern Denmark, Lillebaelt Hospital, Middelfart from 1 January to 31 December 2017 will be identified in the National Patient Register. ${ }^{26}$

Criteria for patient inclusion

1. Included in the SpineData registry.

2. $>60$ years.

3. International Statistical Classification of Diseases, 10th Revision (ICD-10) diagnosis of degenerative lumbar spinal stenosis registered in the nationwide patient registry between 1 January and 31 December 2017 , that is, including central stenosis, foraminal stenosis as 


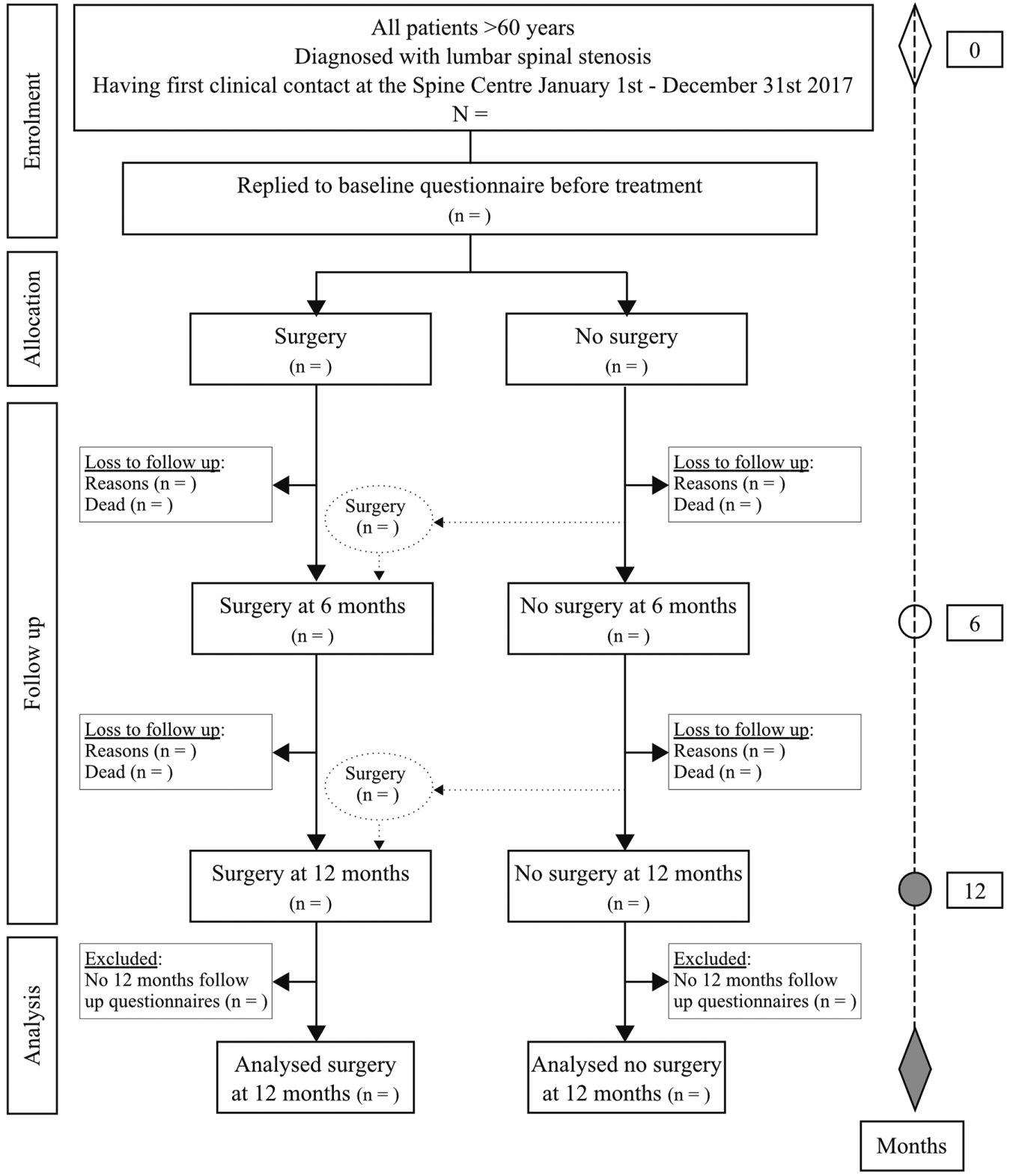

Figure 1 Study flow diagram with enrolment and follow-up.

well as stenosis and spondylolisthesis or spondylodesis combined (DM480, DM996, DM431, DM472).

4. Gives consent to use patient-reported data for research purposes.

\section{Outcome measures}

\section{Primary outcome}

Primary outcome is change in physical function score between baseline and 12-month follow-up measured with Zurich Claudication Questionnaire (ZCQ). The questionnaire has good psychometric properties, is widely used and is recommended in outcome assessment of patients with lumbar spinal stenosis. ${ }^{27} 28$ It has three domains (scales): physical function, symptom severity and patient satisfaction with surgery. The satisfaction subscale is omitted in this study, as we include a non-surgical group. The Physical Function Scale contains five items (questions) designed to specifically assess walking capacity in patients with lumbar spinal stenosis, ${ }^{29}$ and the Symptom Severity Scale has seven items. Scores are calculated as the unweighted mean of all answered items and range from 1 to 4 (physical function) or 1 to 5 (symptom severity). Lower scores indicate less disability. The ZCQ score is considered to represent a 'successful improvement' when the subscales are judged as 'success'. ${ }^{30}$ Minimal clinically important difference in mean change is 0.52 on the Physical Function Scale and 0.48 on the Symptom Severity Scale. ${ }^{27}$

\section{Secondary outcomes}

Secondary outcomes are change in score between baseline and 12-month follow-up in:

1. Symptom Severity Score on the ZCQ. ${ }^{27}$

2. Pain-related physical function on the Oswestry Disability Index (ODI). ${ }^{31}$ 
3. HRQL on the EuroQol 5-dimension 3-level (EQ-5D$3 \mathrm{~L}){ }^{32}$

4. General self-efficacy on the General Self Efficacy Scale (GSE). ${ }^{33}$

The ODI assesses pain-related physical functioning in spinal disorders. ${ }^{34}{ }^{35}$ It has been tested extensively, has good psychometric properties and is applicable in a wide variety of settings. ${ }^{3436}$ The ODI contains 10 questions about how back or leg pain affects the ability to manage everyday life. These are summarised to a score ranging from 0 to 100 . Higher scores reflect worse pain and disability.

EQ-5D-3L is a widely used generic measure of HRQL that is considered valid and responsive for patients with chronic low back pain. ${ }^{37}$ It evaluates five dimensions: mobility, self-care, usual activities, pain/discomfort and anxiety/depression, each with three levels of severity. The resulting health states can be converted into a single summary index with a total score ranging from -0.6 to 1 , where 1 corresponds to perfect health. The EQ-5D-3L includes an EQ Visual Analogue Scale from 0 to 100 for respondents' self-rated health. ${ }^{38}$ Higher scores reflect better HRQL.

The GSE measures perceived self-efficacy ${ }^{33}$ and is used in patients with chronic pain. ${ }^{39}$ Higher scores reflect higher self-efficacy.

All questionnaires will either have been validated in Danish or validated in its original language and translated into Danish following scientific standards. ${ }^{40}$

\section{Potential prognostic and extrinsic factors}

The study explores prognostic factors for outcome related to treatment allocation, back and leg pain intensity, duration of symptoms, smoking, comorbidity, anxiety/ depression, previous spinal surgery, pretreatment function, self-rated health, income, general self-efficacy and MRI-graded severity. Pain intensity is measured using the 11-point Numerical Pain Rating Scale. ${ }^{35}$ Comorbidity is assessed using the Charlson Comorbidity Index (ie, including diabetes, cardiovascular and pulmonary comorbidity). ${ }^{41}$ MRI-graded severity is estimated using the classification described by Ishimoto $e t ~ a l .^{4}$

Data on age, sex, height, weight, cohabitation status, work status, level of education, socioeconomic classification, use of primary healthcare services, prior hospital admissions and use of prescriptive analgesic medicine will be collected to control for potential confounding, mediating or moderating effects.

\section{Data collection}

Data on age, sex, cohabitation, ICD-10 diagnosis, treatment, previous spinal surgery, prior hospital admissions, hospital department, work status, use of prescriptive analgesic medicine, income, socioeconomic classification, level of education and number of consultations in primary healthcare centres will be collected from nationwide registers. ${ }^{24}$
At the first clinical contact, patients complete the baseline questionnaire of the SpineData clinical registry which includes questions covering all the health components of WHO's International Classification of Functioning, Disability and Health. ${ }^{42}$ In addition to the outcome measures, information about body mass index (BMI), back and leg pain intensity, duration of symptoms and smoking is available in the SpineData registry. ${ }^{25}$ Wherever possible, questions and questionnaires have been based on evidence of their role in the diagnosis, prognosis or treatment of spinal pain. ${ }^{25}$ Patients complete the questionnaires without assistance from clinicians or administrative personnel.

For patients with diagnostic MRIs performed at Lillebaelt Hospital, radiologists use a standardised protocol classifying mild, moderate or severe central stenosis as described by Ishimoto et al. ${ }^{4}$ MRI descriptions are available in hospital medical records.

The data collected are depicted in table 1 .

\section{Interventions}

Surgical management (exposed)

Patients receiving surgical treatment undergo various types of posterior decompressive surgery. Most common are decompression only (ie, laminectomy or microdecompression) and decompression plus arthrodesis. ${ }^{43}$ Surgical procedure is determined solely by the surgeon and is recorded in the National Patient Register. ${ }^{26}$

\section{Non-surgical management (unexposed)}

Patients managing lumbar spinal stenosis non-surgically are either referred to rehabilitation at a primary healthcare centre or referred back to their general practitioner for treatment. Treatment may include physiotherapy, chiropractic treatment, lifestyle changes and/ or pain management. Postsurgical patients may also be referred to rehabilitation at a primary healthcare centre. Use of healthcare services in primary care is recorded in the National Health Service Register, ${ }^{44}$ while analgesic medicine is available through the National Prescription Registry. ${ }^{45}$

\section{Patient and public involvement}

This study follows the European League Against Rheumatism recommendations for the inclusion of patient research partners in scientific projects. ${ }^{46}$ It is designed with assistance from three Danish patient representatives, Anna Karen Guldager Rüsz, Tove Theilmann Petersen and Peter Christian Christensen. All are diagnosed with lumbar spinal stenosis and were selected in connection with routine care and participation in a multidisciplinary rehabilitation programme for patients with lumbar spinal stenosis. They have participated in discussions about the relevance and purpose of the study and will contribute with comments on the patient information about the study findings.

Choice of outcome measures was discussed with the patients after selection in collaboration with healthcare 
Table 1 Data collected, including primary endpoint

\begin{tabular}{|c|c|c|c|}
\hline Variable & Baseline & 6 months & 12 months \\
\hline \multicolumn{4}{|l|}{ Demographic factors } \\
\hline Age & $\mathrm{X}$ & & \\
\hline Height & $\mathrm{X}$ & & \\
\hline Weight & $x$ & & \\
\hline \multicolumn{4}{|l|}{ Socioeconomic factors } \\
\hline Income & $\mathrm{X}$ & & \\
\hline Socioeconomic classification & $x$ & & \\
\hline Work status & $\mathrm{X}$ & & \\
\hline Highest level of education completed & $x$ & & \\
\hline Previous spinal surgery & $x$ & & \\
\hline No of consultations in primary healthcare centres & $x$ & $x$ & $\mathrm{X}$ \\
\hline No of hospital admissions & $x$ & $x$ & $x$ \\
\hline Daily dosage of prescriptive analgesic medicine & $x$ & $x$ & $x$ \\
\hline Hospital department & $\mathrm{X}$ & $x$ & $x$ \\
\hline \multicolumn{4}{|l|}{ Symptom and pain-related factors } \\
\hline Duration of symptoms & $\mathrm{X}$ & & \\
\hline Low back pain intensity, NPRS & $x$ & $x$ & $x$ \\
\hline Leg pain intensity, NPRS & $x$ & $x$ & $x$ \\
\hline General self-efficacy, GSE & $\mathrm{x}$ & $x$ & $\mathrm{x}$ \\
\hline Self-described health status, EQ-5D-3L & $x$ & $x$ & $X$ \\
\hline Self-rated health-related quality of life, EQ VAS (0-100) & $\mathrm{x}$ & $x$ & $\mathrm{x}$ \\
\hline Anxiety/depression, item 5, EQ-5D-3L & $\mathrm{x}$ & $x$ & $\mathrm{X}$ \\
\hline \multicolumn{4}{|l|}{ Clinical findings } \\
\hline ICD-10 diagnosis code (DM480, DM996, DM431, DM472) & $\mathrm{X}$ & & \\
\hline MRI findings (severity of central stenosis) & $\mathrm{X}$ & & \\
\hline
\end{tabular}

*Primary endpoint.

EQ-5D-3L, EuroQol 5-dimension 3-level; EQ VAS, EuroQoL Visual Analogue Scale; GSE, General Self-Efficacy Scale; ICD-10, International Statistical Classification of Diseases and Related Health Problems 10th revision; NPRS, Numerical Pain Rating Scale; ODI, Oswestry Disability Index; ZCQ, Zurich Claudication Questionnaire.

professionals with extensive clinical experience in diagnosing, goal setting, decision-making and treating patients with lumbar spinal stenosis and including patient preferences into this process.

A lay summary of the study results will be disseminated through patient groups and online fora to raise awareness of the study and future research areas.

\section{Statistical analyses}

We anticipate that over 12 months, the SpineData registry will collect information on 2500-3000 baseline episodes for consenting patients over 60 years with low back pain, of which 300 patients are expected to undergo surgery for lumbar spinal stenosis. ${ }^{47}$ Assuming equal distribution of patients treated surgically and non-surgically after 1 year, 


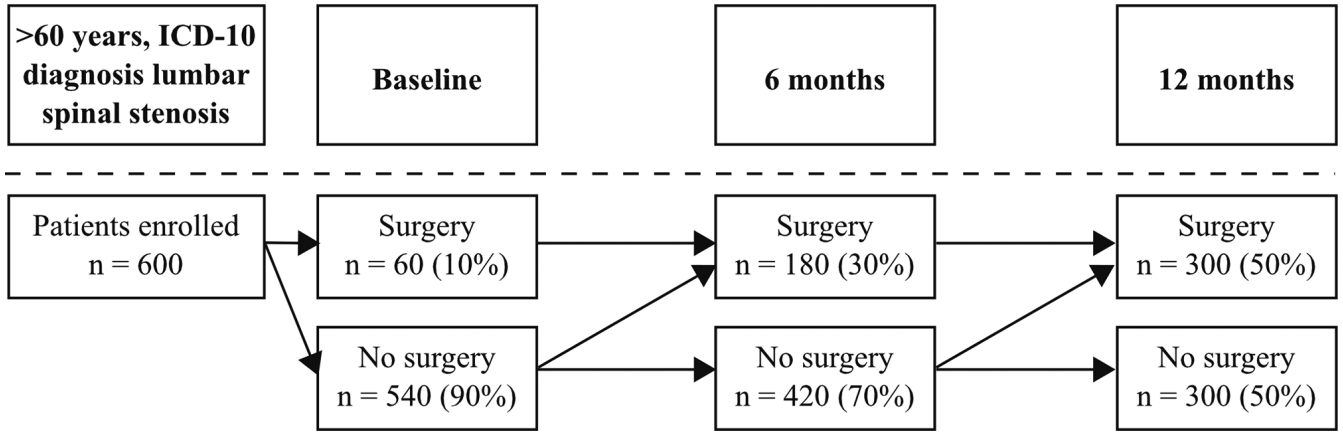

Figure 2 Expected distribution of enrolled patients. ICD-10, International Statistical Classification of Diseases, 10th Revision.

we guesstimate to enrol 600 patients to the lumbar spinal stenosis cohort.

To determine if this cohort size will be sufficient to achieve $\geq 80 \%$ probability of detecting a statistically significant difference in primary outcome between groups, an a priori power analysis was performed.

An enrolment flow and distribution of 600 patients in a balanced design (figure 2) will have a power of 0.956 for a two-sample pooled t-test of a normal mean difference with a two-sided significance level of 0.05 ( $\mathrm{p} \leq 0.05)$, assuming a common SD of 1 point, to detect a mean difference of 0.3 points on the Physical Function Scale.

With the same assumptions, in case of missing data, a balanced design with total sample size of 400 will have a power of 0.849 to detect a mean difference of 0.3 points on the Physical Function Scale.

Even if we are to test for equivalence between surgical and non-surgical treatment, a total sample size of 400 in a balanced design would yield a power of 0.999 for two one-sided tests for additive equivalence of two-sample normal means with bounds -0.25 and 0.25 for the mean difference and a significance level of 0.05 ( $\mathrm{p} \leq 0.05)$, assuming a mean difference of 0 points and a common SD of 0.5 points on the Physical Function Scale.

Following recommendations for the development of a multivariable prognostic model of 50 events for the first covariate and adding 10-20 events required for each prognostic variable, ${ }^{48}$ we would be able to perform a multivariable analysis for potential prognostic factors for outcome with approximately 30 variables in a cohort of 600 patients.

Patient characteristics will be summarised using descriptive statistics, that is, as means with SDs (or medians with IQR) and percentages. Variables and outcome data will be compared between patients who undergo surgery for lumbar spinal stenosis (exposed) and patients who have non-surgical management (unexposed). Data will be analysed in analogy to a randomised trial ${ }^{49}$ while realising, however, that this comparative effectiveness study with repeated measures is not randomly assigning individuals to treatment groups.

The crude statistical model will include two fixed effect factors of group and time and the interaction between them. This model will aim to describe the longitudinal progress (trajectories) for the two groups and subsequently adjust for potentially confounding variables as a consequence of patients not being randomly assigned to the two groups (adjusted model). Random effects result from variation between and within participants; anticipating that measures on the same participant at different times are correlated, and that measures taken close together in time are more highly correlated than measures taken far apart. Observations on different participants are assumed to be independent. In the statistical analyses, the following 'candidate confounding variables' will be considered for statistical adjustment: age, level of education, socioeconomic classification, BMI, comorbidity, use of primary healthcare services, prior hospital admissions and use of prescriptive analgesic medicine.

One of the primary distinguishing features of analysis of repeated measures data is the need to accommodate the covariation of the measures on the same sampling unit. For the choice of covariance structure, we will use graphical techniques, numerical comparisons of covariance estimates and indices of goodness of fit. After the covariance is satisfactorily modelled, the estimated covariance matrix is used to compute generalised least squares estimates of fixed effects of treatments and time.

The statistical model will also compare the study population with those withdrawing or crossing over to surgery. Multivariable analyses will be applied to derive models adjusting for multiple factors. For the purpose of the prespecified analyses, we will consider the 'data as available' to constitute the primary analysis population. As indicated below, this cohort of observed patients will allow patients to 'change group' between baseline and the 12-month assessment and thus violate the intentionto-treat principle.

\section{Missing data}

Missing data can threaten the validity of longitudinal clinical studies. In the absence of an analytical approach that can ensure unbiased estimates of treatment effects with missing data, we will aim to minimise the amount of missing data.

We will distinguish between treatment discontinuation (ie, switching between groups) and missing outcome data. $^{50}$ Data collection is often stopped after treatment discontinuation, but we will attempt to continue recording outcome data on individuals who discontinue/ 
switch from the initial treatment group or who miss clinic visits. While missing outcome data from individuals who continue treatment but are lost to follow-up is a common cause of missing data, ${ }^{50}$ group switching is better viewed as a form of non-compliance and will be treated using ideas from the causal literature on non-compliance. ${ }^{51}$

We consider methods of analysis that are model based to be superior to available-case, complete-case analysis or single-imputation methods (eg, the last observation carried forward). As the proportion of missing data may raise questions about the validity of preferred methods such as model-based imputation, we will perform protocolised sensitivity analyses to determine whether the conclusions are sensitive to assumptions about the reasons for missing data.

\section{Analysis populations}

The full analysis set is as close as possible to the ideal population implied by the intention-to-treat principle. However, missing data within participants can present serious problems depending on the amount, cause and pattern of missing data, particularly in a non-randomised study design. The 'as observed population', allowing for switch between groups, will be compared with two alternative populations, (1) the intention-to-treat population in which data collected for the individual patient stay in the initial group and (2) the per-protocol population in which only those without 'protocol violations' will be included; see below for definitions:

As-observed population (available-case analysis): This is based on the 'full analysis set', being all the data available at baseline and no replacement for missing data, where patients can legitimately change group according to what is observed, that is, patients can end up with (1) only surgery, (2) only non-surgical management or (3) a secondary switch to accept surgery.

Intention-to-treat population: The underlying principle is that the effect of a treatment policy, such as non-surgical management, can be best assessed by basing the analysis on the intention to treat an individual, hence the planned treatment regimen rather than the actual treatment given. Patients initially allocated to a specific treatment group should thus be followed up, assessed and analysed as members of that group irrespective of their compliance to the planned course of treatment. Initial non-surgical patients thus remain in that group even if they subsequently have surgery, while initial surgical patients remain in that group even if they use postsurgical rehabilitation.

Per-protocol population: Here, the analysis is restricted to the subset of patients who sufficiently comply with the protocol that their data are likely to exhibit the effects of treatment. The per-protocol non-surgical population will thus include all patients in the intention-to-treat population but will exclude those crossing over from non-surgical management to surgery. The per-protocol surgical population will include all patients in the intention-totreat population but will exclude patients having surgery less than 3 months prior to completing the 12-month follow-up. Pain and physical impairment are expected to increase immediately after surgery, and patients undergoing surgery for lumbar spinal stenosis at the Spine Centre of Southern Denmark are advised to restrict their activities in a postsurgical recovery period of up to 12 weeks. Patient-reported outcome recorded during this period is thus assumed to be less relevant for comparison to non-surgical patients.

The analyses will be performed using STATA (V.15.1, StataCorp) and in collaboration with a statistical expert.

\section{ETHICS AND DISSEMINATION}

The study will be performed according to the Declaration of Helsinki, ${ }^{52}$ the European Code of Conduct for Research Integrity $^{53}$ and the Danish Code of Conduct for Research Integrity. ${ }^{54}$ The study has been notified to the Danish Data Protection Agency ${ }^{56}$ (17/30636), and permission to extract data from hospital records will be obtained from the Danish Patient Safety Authority. ${ }^{57}$ Consent to use patient-reported information from the SpineData registry is obtained electronically prior to patients completing the questionnaires. Patients who do not consent will not be included.

Findings will be disseminated in peer-reviewed publications and presented at national and international conferences following guidance from the Strengthening the Reporting of Observational Studies in Epidemiology ${ }^{58}$ and Prognosis Research Strategy ${ }^{59}$ statements. Potential sources of bias will be addressed using Risk of Bias in Non-randomised Studies of Interventions. ${ }^{49}$

\section{DISCUSSION}

This article presents a protocol for an observational study designed to investigate the course of treatment by comparing the effectiveness of surgical and non-surgical management in patients with lumbar spinal stenosis and identifying prognostic factors for outcome. Prospectively registered data on health and social variables and patient-reported outcome are collected from national registers, the SpineData clinical registry of people with chronic back pain and hospital medical records.

The main limitation of this study is that analyses are not based on randomised treatment assignments, meaning that selection bias is a concern. Patient characteristics not accounted for in this study are likely to influence the decision whether to choose surgery or not. The study would thus benefit from patient groups being more similar with only the intervention differing between the groups.

We aim to strengthen the study results by collecting data prospectively, by having a large sample size (ie, 'guesstimated' from assumptions of expected patient numbers and their distribution across treatment groups), and by linking patient-reported outcome to data from national registers. We are aware that lost to follow-up could be a problem. Data from the clinical SpineData registry may 
only be $50 \%$ at 6 months and $35 \%$ at 12 months, and the proportion of MRI descriptions available in hospital medical records is unknown. To minimise missing data, an independent administrative assistant will send out a reminder, including a paper version of the questionnaires, to patients who do not respond to the 12-month follow-up.

While we do not know the exact treatment modalities in the non-surgical group, we are not comparing outcome of surgery to any specific non-surgical treatment.

This study will assess outcome from non-surgical management and will provide knowledge about factors that can predict outcome in surgical and non-surgical management of lumbar spinal stenosis. This should help clinicians in guiding patients when choosing surgical or non-surgical treatment for lumbar spinal stenosis, and researchers in selecting variables of interest in future randomised controlled trials comparing the effect of the two management options.

\section{Author affiliations}

${ }^{1}$ Spine Centre of Southern Denmark, Sygehus Lillebalt Middelfart Sygehus, Middelfart, Denmark

${ }^{2}$ Department of Regional Health Research, Faculty of Health Services, University of Southern Denmark, Odense, Denmark

${ }^{3}$ Musculoskeletal Statistics Unit, the Parker Institute, Bispebjerg and Frederiksberg Hospital, Copenhagen, Denmark

${ }^{4}$ University College South Denmark, Esbjerg-Haderslev, Denmark

${ }^{5}$ Department of Public Health, Centre for Rehabilitation Research, Section for Clinical Social Medicine and Rehabilitation, Faculty of Health, Aarhus University, Aarhus, Denmark

${ }^{6}$ DEFACTUM, Central Denmark Region, Aarhus, Denmark

${ }^{7}$ Department of Rheumatology, Odense University Hospital, Odense, Denmark

Acknowledgements The authors wish to thank patient research partners Anna Karen Guldager Rüsz, Peter Christian Christensen and Tove Theilmann Petersen for their help in this study. The authors thank Claire Gudex, assistant professor at the Department of Clinical Research, University of Southern Denmark for proofreading of the final draft.

Contributors $\mathrm{HAB}$ and BS-C conceived the study. All authors participated in the study design and the preliminary analysis plan. $\mathrm{HAB}$ constructed the first draft of the manuscript. All authors contributed to writing the manuscript. TM contributed substantially to writing the section on outcome measures. RC contributed substantially to the power analysis calculations and in writing the statistical analyses section. BS-C contributed substantially to writing the introduction and the section on data collection. All authors participated in critical scrutinising and revision of the manuscript and approved the final version.

Funding The authors have not declared a specific grant for this research from any funding agency in the public, commercial or not-for-profit sectors.

Competing interests TM and BS-C have nothing to disclose. $\mathrm{HAB}$ reports unrestricted grants from the University of Southern Denmark, the Region of Southern Denmark, the University College of Southern Denmark and non-financial support from Spine Centre of Southern Denmark, during the conduct of the study. $\mathrm{RC}$ reports non-financial support from Board membership, grants from Consultancy (AbbVie, Amgen, Axellus A/S, Bristol-Myers Squibb, Cambridge Weight Plan, Celgene, Eli Lilly, Hospira, MSD, Norpharma, Novartis, Orkla Health, Pfizer, Roche, Sobi, Takeda), personal fees from Employment (Research Unit for Musculoskeletal Function and Physiotherapy, Institute of Sports Science and Clinical Biomechanics, University of Southern Denmark), non-financial support from Expert testimony, grants from grants/grants pending (Axellus A/S, AbbVie, Cambridge Weight Plan, Janssen, MSD, Mundipharma, Novartis and Roche), grants from payment for lectures including service on speakers bureaus (Abbott, Amgen, Axellus, Bayer HealthCare Pharmaceuticals, Biogen Idec, Bristol-Myers Squibb, Cambridge Weight Plan, Ipsen, Janssen, Laboratoires Expanscience, MSD, Mundipharma, Norpharma, Novartis, Pfizer, Roche, Rottapharm-Madaus, Sobi and Wyeth), grants from payment for manuscript preparation (Axellus, Bristol-Myers Squibb and Cambridge Weight Plan, Aleris-Hamlet (via Norpharma)), non-financial support from Patents (planned, pending or issued), non-financial support from Royalties, grants from payment for development of educational presentations (Bristol-Myers Squibb, MSD, Pfizer), nonfinancial support from stock/stock options, grants from travel/accommodations/ meeting expenses unrelated to activities listed (Abbott, AbbVie, Axellus, Biogen, Bristol-Myers Squibb, Cambridge Weight Plan, Celgene, Laboratoires Expanscience, Norpharma, Novartis, Pfizer, Roche, Rottapharm-Madaus and Wyeth), non-financial support from Other (err on the side of full disclosure), outside the submitted work and is involved in many healthcare initiatives and research that could benefit from wide uptake of this publication (including Cochrane, OMERACT, IDEOM, RADS and the GRADE Working Group). Musculoskeletal Statistics Unit, The Parker Institute is grateful for the financial support received from public and private foundations, companies and private individuals over the years. The Parker Institute is supported by a core grant from the Oak Foundation; The Oak Foundation is a group of philanthropic organisations that, since its establishment in 1983, has given grants to not-for-profit organisations around the world.

\section{Patient consent for publication Not required.}

Ethics approval A review by the Regional Committees on Health Research Ethics for Southern Denmark concluded that ethical approval was not required for this study according to the Danish Act on Research Ethics Review of Health Research Projects (S-20172000-200).

Provenance and peer review Not commissioned; externally peer reviewed.

Open access This is an open access article distributed in accordance with the Creative Commons Attribution Non Commercial (CC BY-NC 4.0) license, which permits others to distribute, remix, adapt, build upon this work non-commercially, and license their derivative works on different terms, provided the original work is properly cited, appropriate credit is given, any changes made indicated, and the use is non-commercial. See: http://creativecommons.org/licenses/by-nc/4.0/.

\section{REFERENCES}

1. Zaina F, Tomkins-Lane C, Carragee E, et al. Surgical versus nonsurgical treatment for lumbar spinal stenosis. Cochrane Database Syst Rev 2016;1:CD010264.

2. Lurie J, Tomkins-Lane C. Management of lumbar spinal stenosis. BMJ 2016;352:h6234.

3. Melancia JL, Francisco AF, Antunes JL, et al. Spinal stenosis. Handb Clin Neurol 2014;119:541-9.

4. Ishimoto $\mathrm{Y}$, Yoshimura N, Muraki S, et al. Associations between radiographic lumbar spinal stenosis and clinical symptoms in the general population: the Wakayama Spine Study. Osteoarthritis Cartilage 2013;21:783-8.

5. Minamide A, Yoshida M, Maio K. The natural clinical course of lumbar spinal stenosis: a longitudinal cohort study over a minimum of 10years. Journal of Orthopaedic Science 2013;18:693-8.

6. Whitman JM, Flynn TW, Childs JD, et al. A comparison between two physical therapy treatment programs for patients with lumbar spinal stenosis: a randomized clinical trial. Spine 2006;31:2541-9.

7. Ammendolia C, Stuber KJ, Rok E, et al. Nonoperative treatment for lumbar spinal stenosis with neurogenic claudication. Cochrane Database Syst Rev 2013;8:CD010712.

8. Zweig T, Enke J, Mannion AF, et al. Is the duration of pre-operative conservative treatment associated with the clinical outcome following surgical decompression for lumbar spinal stenosis? A study based on the Spine Tango Registry. Eur Spine J 2017;26.

9. Kovacs FM, Urrútia G, Alarcón JD. Surgery versus conservative treatment for symptomatic lumbar spinal stenosis: a systematic review of randomized controlled trials. Spine 2011;36:E1335-51.

10. Deyo RA, Mirza SK, Martin BI, et al. Trends, major medical complications, and charges associated with surgery for lumbar spinal stenosis in older adults. JAMA 2010;303:1259-65.

11. Giannadakis $C$, Solheim $O$, Jakola AS, et al. Surgery for lumbar spinal stenosis in individuals aged 80 and older: a multicenter observational study. J Am Geriatr Soc 2016;64:2011-8.

12. Madsbu MA, Solberg TK, Salvesen $\varnothing$, et al. Surgery for herniated lumbar disk in individuals 65 years of age or older: a multicenter observational study. JAMA Surg 2017;152:503-6.

13. Kleinstück FS, Grob D, Lattig F, et al. The influence of preoperative back pain on the outcome of lumbar decompression surgery. Spine 2009;34:1198-203.

14. Gulati S, Nordseth T, Nerland US, et al. Does daily tobacco smoking affect outcomes after microdecompression for degenerative central 
lumbar spinal stenosis? - A multicenter observational registry-based study. Acta Neurochir 2015;157:1157-64.

15. Nerland US, Jakola AS, Giannadakis C, et al. The risk of getting worse: predictors of deterioration after decompressive surgery for lumbar spinal stenosis: a multicenter observational study. World Neurosurg 2015;84:1095-102.

16. Aalto TJ, Malmivaara A, Kovacs F, et al. Preoperative predictors for postoperative clinical outcome in lumbar spinal stenosis: systematic review. Spine 2006;31:E648-E663.

17. Jonker AA, Comijs HC, Knipscheer KC, et al. Benefits for elders with vulnerable health from the Chronic Disease Self-management Program (CDSMP) at short and longer term. BMC Geriatr 2015;15:101.

18. Moojen WA, Schenck CD, Lycklama À Nijeholt GJ, et al. Preoperative $\mathrm{MRI}$ in Patients with intermittent neurogenic claudication: relevance for diagnosis and prognosis. Spine 2018;43:348-355.

19. Weber C, Giannadakis C, Rao V, et al. Is There an association between radiological severity of lumbar spinal stenosis and disability, pain, or surgical outcome?: a multicenter observational study. Spine 2016;41:E78-83.

20. Schneider MJ, Terhorst L, Murphy D, et al. Exploratory analysis of clinical predictors of outcomes of nonsurgical treatment in patients with lumbar spinal stenosis. J Manipulative Physiol Ther 2016;39:88-94.

21. Kurd MF, Lurie JD, Zhao W, et al. Predictors of treatment choice in lumbar spinal stenosis: a spine patient outcomes research trial study. Spine 2012;37:1702-7.

22. Delitto A, Piva SR, Moore CG, et al. Surgery versus nonsurgical treatment of lumbar spinal stenosis. Ann Intern Med 2015;163:397-8.

23. Fiore LD, Lavori PW. Integrating randomized comparative effectiveness research with patient care. $N$ Engl J Med 2016;374:2152-8.

24. Thygesen LC, Daasnes C, Thaulow I, et al. Introduction to Danish (nationwide) registers on health and social issues: structure, access, legislation, and archiving. Scand J Public Health 2011;39:12-16.

25. Kent P, Kongsted A, Jensen TS, et al. SpineData - a Danish clinical registry of people with chronic back pain. Clin Epidemiol 2015;7:369-80.

26. Lynge E, Sandegaard JL, Rebolj M. The Danish National Patient Register. Scand J Public Health 2011;39:30-3.

27. Stucki G, Daltroy L, Liang MH, et al. Measurement properties of a self-administered outcome measure in lumbar spinal stenosis. Spine 1996;21:796-803.

28. Tomkins-Lane CC, Battié MC. Validity and reproducibility of selfreport measures of walking capacity in lumbar spinal stenosis. Spine 2010;35:2097-102.

29. Tomkins-Lane CC, Battié MC, Macedo LG. Longitudinal construct validity and responsiveness of measures of walking capacity in individuals with lumbar spinal stenosis. Spine J 2014;14:1936-43.

30. Tuli SK, Yerby SA, Katz JN. Methodological approaches to developing criteria for improvement in lumbar spinal stenosis surgery. Spine 2006;31:1276-80.

31. Lauridsen $\mathrm{HH}$, Hartvigsen J, Manniche C, et al. Danish version of the oswestry disability index for patients with low back pain. Part 1: cross-cultural adaptation, reliability and validity in two different populations. Eur Spine J 2006;15:1705-16.

32. Obradovic M, Lal A, Liedgens $\mathrm{H}$. Validity and responsiveness of EuroQol-5 dimension (EQ-5D) versus Short Form-6 dimension (SF-6D) questionnaire in chronic pain. Health Qual Life Outcomes 2013;11:110.

33. Luszczynska A, Scholz U, Schwarzer R. The general self-efficacy scale: multicultural validation studies. J Psychol 2005;139:439-57.

34. Fairbank JC, Pynsent PB. The Oswestry Disability Index. Spine 2000;25:2940-53.

35. Chiarotto A, Boers M, Deyo RA, et al. Core outcome measurement instruments for clinical trials in non-specific low back pain. Pain (Published 24 November 2017).

36. Nerland US, Jakola AS, Solheim O, et al. Comparative effectiveness of microdecompression and laminectomy for central lumbar spinal stenosis: study protocol for an observational study. BMJ Open 2014;4:e004651.
37. Soer R, Reneman MF, Speijer BL, et al. Clinimetric properties of the EuroQol-5D in patients with chronic low back pain. Spine $J$ 2012;12:1035-9.

38. EQ-5D. EQ-5D User Guides - EQ-5D. https://euroqol.org/ publications/user-guides/ (Accessed $10 \mathrm{Jul}$ 2017).

39. The General Self-Efficacy Scale (GSE). http://userpage.fu-berlin.de/ health/engscal.htm (Accessed $10 \mathrm{Jul}$ 2017).

40. Acquadro C, Conway K, Hareendran A, et al. Literature review of methods to translate health-related quality of life questionnaires for use in multinational clinical trials. Value Health 2008;11:509-21.

41. Charlson ME, Pompei P, Ales KL, et al. A new method of classifying prognostic comorbidity in longitudinal studies: development and validation. J Chronic Dis 1987;40:373-83.

42. WHO. International Classification of Functioning, Disability and Health (ICF): WHO, 2017.

43. Lønne G, Fritzell P, Hägg $O$, et al. Lumbar spinal stenosis: comparison of surgical practice variation and clinical outcome in three national spine registries. Spine $J 2018$ (Published 21 May 2018).

44. Andersen JS, Olivarius NF, Krasnik A. The Danish National Health Service Register. Scand J Public Health 2011;39:34-7.

45. Kildemoes HW, Sørensen HT, Hallas J. The Danish National prescription registry. Scand J Public Health 2011;39:38-41.

46. de Wit MP, Berlo SE, Aanerud GJ, et al. European League against rheumatism recommendations for the inclusion of patient representatives in scientific projects. Ann Rheum Dis 2011;70:722-6.

47. drks-webmaster. Danespine årsrapport 2016 - Dansk Rygkirurgisk Selskab. 2016 http://drks.ortopaedi.dk/danespine-aarsrapport-2016/ (Accessed 16 Jan 2018).

48. Harrell FE, Lee KL, Mark DB. Multivariable prognostic models: issues in developing models, evaluating assumptions and adequacy, and measuring and reducing errors. Stat Med 1996;15:361-87.

49. Sterne JA, Hernán MA, Reeves BC, et al. ROBINS-I: a tool for assessing risk of bias in non-randomised studies of interventions. BMJ 2016;355:i4919.

50. Vickers AJ, Altman DG. Statistics notes: missing outcomes in randomised trials. BMJ 2013;346:f3438.

51. Little R, Kang S. Intention-to-treat analysis with treatment discontinuation and missing data in clinical trials. Stat Med 2015;34:2381-90.

52. WMA - The World Medical Association. WMA Declaration of Helsinki - Ethical Principles for Medical Research Involving Human Subjects. https://www.wma.net/policies-post/wma-declaration-of-helsinkiethical-principles-for-medical-research-involving-human-subjects/ (Accessed 5 Jul 2017).

53. Alleaadmin. ALLEA publishes revised edition of The European Code of Conduct for Research Integrity. http://www.allea.org/ allea-publishes-revised-edition-european-code-conduct-researchintegrity/ (Accessed 5 Jul 2017).

54. sbj. The Danish Code of Conduct for Research Integrity Uddannelses- og Forskningsministeriet. http://ufm.dk/publikationer/ 2014/the-danish-code-of-conduct-for-research-integrity (Accessed 5 Jul 2017).

55. Act on Research Ethics Review of Health Research Projects. http:// www.nvk.dk/english/act-on-research (Accessed 5 Jul 2017).

56. Datatilsynet: introduction to the Danish data protection agency. https://www.datatilsynet.dk/english/the-danish-data-protectionagency/introduction-to-the-danish-data-protection-agency/ (Accessed 5 Jul 2017).

57. Videregivelse af patientjournaloplysninger - Styrelsen for Patientsikkerhed. https://stps.dk/da/sundhedsprofessionelle-ogmyndigheder/patientjournaloplysninger/ (Accessed 12 Jul 2017).

58. von Elm E, Altman DG, Egger M, et al. The strengthening the reporting of observational studies in epidemiology (STROBE) statement: guidelines for reporting observational studies. Int J Surg 2014;12:1495-9.

59. Hemingway $\mathrm{H}$, Croft $\mathrm{P}$, Perel $\mathrm{P}$, et al. Prognosis research strategy (PROGRESS) 1: a framework for researching clinical outcomes. BMJ 2013;346:e5595. 